\title{
Fundamental Bandwidth Limitation for Small Antennas on a Platform
}

\author{
Johnson J. H. Wang \\ Wang Electro-Opto Corporation (WEO) \\ 2140 Newmarket Parkway, Suite 110, Marietta, Georgia 30067 USA \\ Email:jjhwang@weo.com
}

\section{INTRODUCTION}

Classical theory on fundamental gain bandwidth limitation for antennas constrained by their electrical size has been extensively examined, and is collectively referred to here as the Chu theory [1]. However, there are major shortcomings and ambiguities in the Chu theory when applied to real world problems, as pointed out recently by this author [2]. One problem is the case of an antenna on a platform, as depicted in Fig. 1, where the antenna is generally inseparable from the transceiver/platform. In fact, in some designs the main radiator is the platform or transceiver, not the antenna per se. Thus, the extent and size of the antenna become ambiguous. Also, the Chu theory is valid only for high $Q$ (Quality factor). With the platform becoming part of it, the antenna's effective size is increased and its $Q$ can be lowered beyond the realm of the Chu theory.

This paper discusses the fundamental bandwidth limitation of small antennas (less than 1 wavelength in extent at the low end of its operating band) mounted on a platform, beginning with a review of the basic concepts and terminology

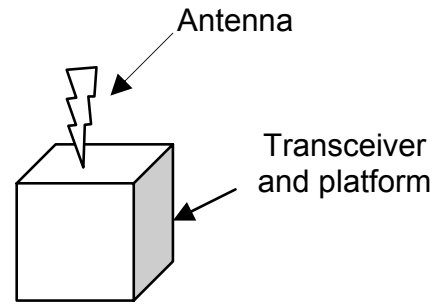

Fig. 1. Antenna with transceiver and on platform. involved.

\section{TWO DEFINITIONS FOR BANDWIDTH}

As background information, we start with the definition of fractional bandwidth, $B_{f}$, given by

$$
B_{f} \equiv \Delta f / f_{\mathrm{c}} \equiv\left(f_{\mathrm{H}}-f_{\mathrm{L}}\right) / f_{\mathrm{c}}=2\left(f_{\mathrm{H}}-f_{\mathrm{L}}\right) /\left(f_{\mathrm{H}}+f_{\mathrm{L}}\right)<200 \%
$$

where $f_{\mathrm{c}}, f_{\mathrm{H}}$, and $f_{\mathrm{L}}$ denote the frequencies at the center, the high end, and the low end of the operating bandwidth, respectively. The fractional bandwidth is clear and precise for narrowband systems.

Note that fractional bandwidth is always less than 200\%; thus it is a muddled scale for broadband systems. For broadband systems, it is desirable and often necessary to adopt the "octaval bandwidth" given by

$$
B_{\mathrm{o}} \equiv f_{\mathrm{H}} / f_{\mathrm{L}}
$$

where the octaval bandwidth $B_{\mathrm{o}}$ is a dimensionless quantity expressed similar to the SWR (e.g., 10 or 10:1), or in octaves. Note that $B_{\mathrm{o}}$ can be infinite while $B_{f}$ is always less than $200 \%$. The octaval bandwidth offers a clear and precise scale for broadband systems.

\section{ISSUES RELATED TO ANTENNAS ON SMALL PLATFORM}

\section{Antenna Size}

Only small antennas (with electrical size less than 1 wavelength) are under consideration here. Due to their small size, these antennas have broad-beamed patterns, which can be classified into two types: directional and omnidirectional.

\section{Resonant Antenna and Traveling-wave (TW) Antenna}

Small antennas used on a platform can be classified into two basic types: resonant antennas and traveling-wave (TW) antennas. The resonant antenna is characterized by a source distribution that can only be matched to free space by resonance mechanism, which is inherently narrowband. 
The only nonresonance antenna known to date is the TW antenna. A TW antenna is characterized by a source distribution in the form of a directional modal wave or current which radiates its energy while traveling along the antenna. As pointed out earlier [3, 4], ultra-wideband as a rule is achieved only by TW antennas.

\section{Conformal Antenna and Nonconformal Antenna and the Issue of Radiation Hazard}

Antennas employed on a small platform can also be classified into two types according to their physical configuration: conformal antennas and nonconformal antennas. A truly conformal antenna contains a conducting surface that can shield the radiating element from the platform.

It is worthwhile to emphasize again that in real world an antenna is rarely an object isolated in space. The antenna's specific extent and size become ambiguous when it is electrically small. This can present a severe problem in measurement. Indeed, there have been many papers containing erroneous measured antenna gain patterns which had largely resulted from radiating connecting cables and test equipment, not the antenna per se.

In addition, radiation hazard from the transmitted RF power becomes a serious issue as a nearby human body becomes part of the platform. In this connection it is worth pointing out that the present SAR (Specific Absorption Rate) standard often masks the danger of "hot spot" as well as other health effects besides simple thermal damage.

Based on considerations in performance and radiation hazard, as discussed, the conformal antenna appears to be the most desirable, and often the only acceptable, choice for antennas in handheld, body-borne, and some other smallplatform applications. Unfortunately, it is difficult for conformal antennas to achieve both broad bandwidth and low profile. So far the only conformal antennas with established multioctave bandwidth known to this author are the SMM (Spiral-Mode Microstrip) antenna [3,4] and the SW (slow-wave) antenna [5].

\section{REVIEW OF CLASSICAL THEORY ON ANTENNA BANDWIDTH LIMITATION AND ITS PITFALLS IN APPLYING TO ANTENNAS ON PLATFORM}

For a free-standing lossless resonant omnidirectional antenna, the maximum achievable octaval bandwidth $\left(B_{\mathrm{o}}\right)$ and the quality factor $(Q)$ as a function of antenna size $k a$, according to the classical Chu theory, are plotted in Fig. 2. Here $a$ is the radius of a sphere enclosing the antenna, and $k$ $=2 \pi / \lambda$ with $\lambda$ being the wavelength at the center frequency $f_{\mathrm{c}}$. The McClean curve [6] is a refinement of the original $\mathrm{Chu}$ formula.

Note, however, that the $\mathrm{Chu}$ theory is only valid for antennas of high $Q$ (quality factor) because it is based on the relationship between $Q$ and bandwidth, which rapidly becomes invalid as $Q$ decreases below 4 .

Note also that the $Q$ of a planar aperture antenna is related to its bandwidth only under certain constraints which cannot be realized [7]. Thus, the Chu theory breaks down for the conformal TW Octaval Bandwidth $B_{o}$ and $Q$ vs $k a$

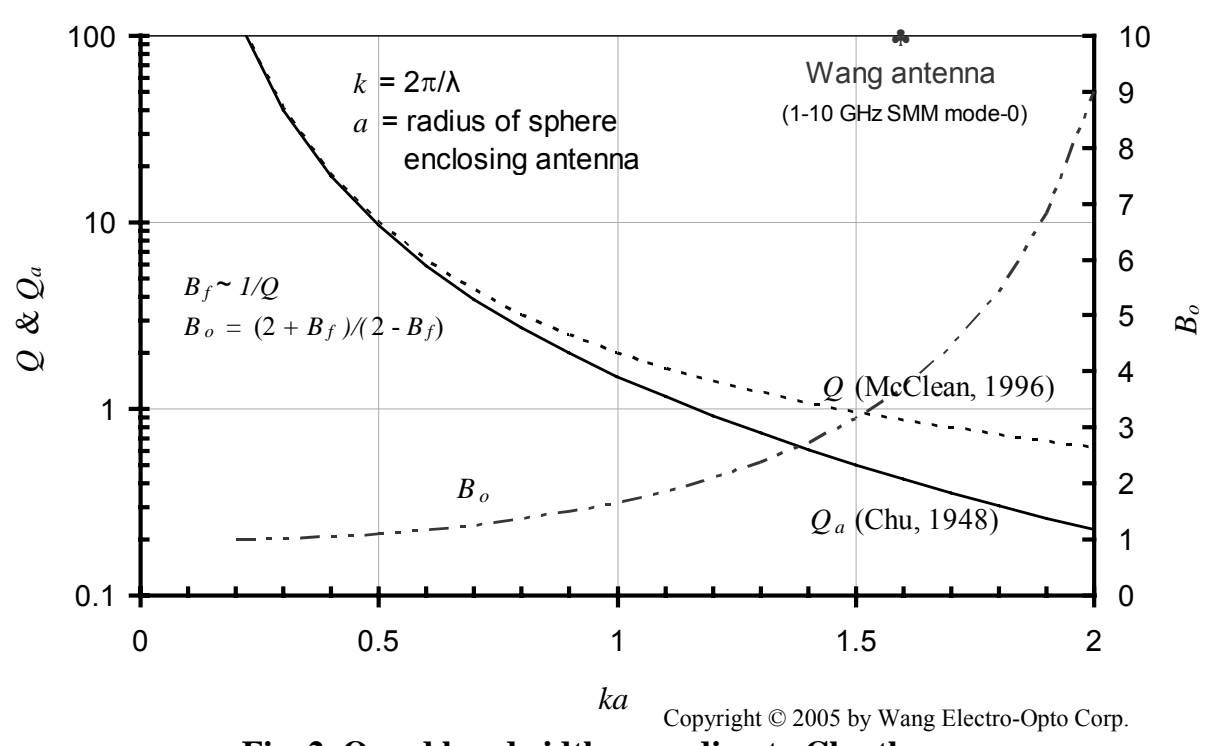

Fig. 2. $\mathrm{Q}$ and bandwidth according to $\mathrm{Chu}$ theory. antenna on a platform, which in essence is a planar aperture antenna. As an example, the bandwidth of the Wang conformal TW antenna [8], as shown in Fig. 2, exceeds the Chu limitation, exhibiting a large octaval bandwidth $B_{o}=$ 10. (The Wang antenna is 6 " in diameter, or $k a=1.6$ at $1 \mathrm{GHz}$, and is mounted on a 12" conducting plate.) 
It must be pointed out that the chart in Fig. 2 should be taken with a grain of salt. First, the bandwidth in the Chu theory is fractional bandwidth, not octaval bandwidth. Second, fractional bandwidth and the Chu theory are tailored for narrow, resonant bandwidth, not appropriate for the ultrawide bandwidth of TW antennas.

To explore the issues deeper, let us examine the recent discussions on impedance, bandwidth, and $Q$ of antennas by Yaghjian and Best [9]. Although their analyses clarify certain issues of interest, including the invalidity of Foster reactance theorem for antennas, they do not address the fundamental bandwidth limitation imposed by antenna size. They merely reveal the $Q$ and thus the bandwidth limitation (yet only the impedance bandwidth) for specific antenna design configurations. Additionally, their inclusion of multiple resonances and antiresonances does not do justice to the " $Q$ " or the bandwidth of the antennas which are not electrically small, even though it reflects proper recognition of a large potential bandwidth. For example, the 3-element Yagi antenna in Fig. 13 of [9] has a linear dimension of 0.24 $\lambda_{\mathrm{L}}$ at $f_{\mathrm{L}}=191 \mathrm{MHz}$, yet shows moderately high $Q(5$ to 20 over 191-700 MHz) in Figs. 15-17. The moderately high $Q$, and the consequential lack of broadbanding potential, are merely due to the specific configuration of this particular design which operates on resonance and antiresonance mechanisms. If a TW antenna is chosen under this size constraint, octaval bandwidth over 10 can be achieved, as has been demonstrated recently [8].

\section{FUNDAMENTAL BANDWIDTH LIMITATION OF SMALL ANTENNAS ON PLATFORM}

\section{Empirical Theory}

An empirical theory on fundamental bandwidth limitation due to size constraints has been developed for small antennas on platform based on existing electromagnetic theory and experimental observation, and is summarized in Table 1. For a lossless narrowband resonance antenna on platform, the Chu theory can be applied with proper inclusion of the platform/transceiver, as discussed earlier. For a small conformable disk-shaped TW antenna, as specified, on a platform, there is no theoretical fundamental limitation on its octaval bandwidth. However, its lower bound of the bandwidth is limited by its size, and its practical octaval bandwidth is finite, being less than 20 . The reduced practical bandwidth limitation is largely due to the TW antenna's radiation property (pattern and polarization), not impedance. The practical impedance bandwidth of the TW antenna can be much larger than 20.

Table 1. Fundamental bandwidth limitation for small antenna $\left(<\lambda_{\mathrm{L}}\right)$ on platform. ( $\lambda_{\mathrm{L}}=$ wavelength at the lower bound of the operating band)

\begin{tabular}{|c|c|c|}
\hline Antenna Type & $\begin{array}{l}\text { Maximum Theoretical } \\
\text { Octaval Bandwidth } B_{0}\end{array}$ & $\begin{array}{c}\text { Maximum Practical } \\
\text { Octaval Bandwidth } B_{0}\end{array}$ \\
\hline $\begin{array}{c}\text { Omnidirectional conformable } \\
\text { TW antenna }\end{array}$ & $\begin{array}{c}B_{\mathrm{o}} \rightarrow \infty \\
\text { if }\left\{\begin{array}{l}\text { antenna radius }>\lambda_{\mathrm{L}} / 4 \\
\text { antenna height }>\lambda_{\mathrm{L}} /(4 \pi)\end{array}\right.\end{array}$ & $<20$ \\
\hline $\begin{array}{l}\text { Directional conformable } \\
\text { TW antenna }\end{array}$ & $\begin{array}{c}B_{\mathrm{o}} \rightarrow \infty \\
\text { if }\left\{\begin{array}{c}\text { antenna radius }>\lambda_{\mathrm{L}} /(2 \pi) \\
\text { antenna height }>\lambda_{\mathrm{L}} /(16 \pi)\end{array}\right.\end{array}$ & $<20$ \\
\hline Resonance antenna & $\begin{array}{l}\text { Chu theory without consideration of } \\
\text { platform/transceiver }\end{array}$ & $\begin{array}{l}\text { Chu theory applied to combined } \\
\text { antenna and platform/transceiver }\end{array}$ \\
\hline
\end{tabular}

Note that the TW antennas must have a low profile to meet user needs, especially at frequencies below, say, $2 \mathrm{GHz}$, and in particular if the platform is small.

\section{Supporting Experiments}

Extensive investigation, in both theory and experiments, has been made by this author for the broadbanding and size reduction of antennas. The summary in Table 1 is of course subject to interpretation and clarification, perhaps more than that for the classical Chu theory; yet it is useful for practical design work. Indeed, recent research in small broadband antennas on platform has led to the observation that a complete solution to this problem is to employ a conformal TW antenna. A bona fide conformal antenna has a conducting ground surface, which can be used to minimize the effect of the platform on antenna radiation and also shield the human body from radiation hazard. Fig. 3 shows four breadboard models developed at WEO. Each model has achieved a broader bandwidth than that constrained 
by the classical Chu theory. Physically, the broader bandwidth benefits enormously from the larger size of the platform as well as the TW radiation mechanism.

In Fig. 3(a), a disk-shaped slow-wave (SW) antenna [5] is mounted on top of the "clam shell" of a cell phone (and would be integrated into it later). Fig. 3(b) shows a helmet antenna where the antenna is hidden inside a kevlar helmet [10]. Fig. 3(c) shows a vest antenna where the antenna is attached to the front and back of a military suit worn by a soldier [11]. (The antenna can be integrated into the military suit or its pouches.) Fig. 3(d)

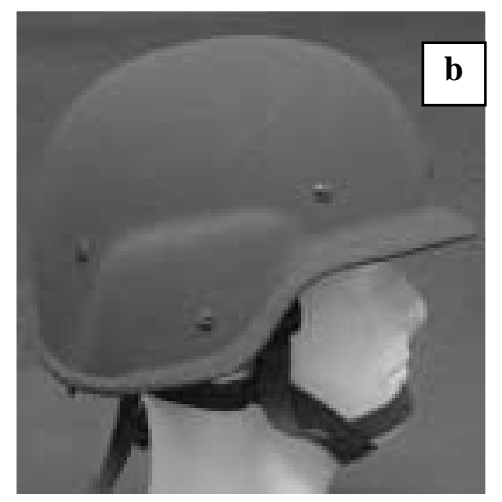
shows a disk-shaped 1-10 GHz antenna [8] which can be readily mounted on the metallic top or bottom surface of a vehicle using the mounting holes shown.

In these designs, the SW antenna [5] or SMM antenna [3, 4] is employed. Both SW and SMM antennas are conformable antennas with a conducting ground plane that can be readily integrated into the surface of the platform, which can be either conducting or nonconducting. As can be seen, a conformal antenna is integrated into, or directly mounted on, each small platform.

\section{CONCLUSIONS}

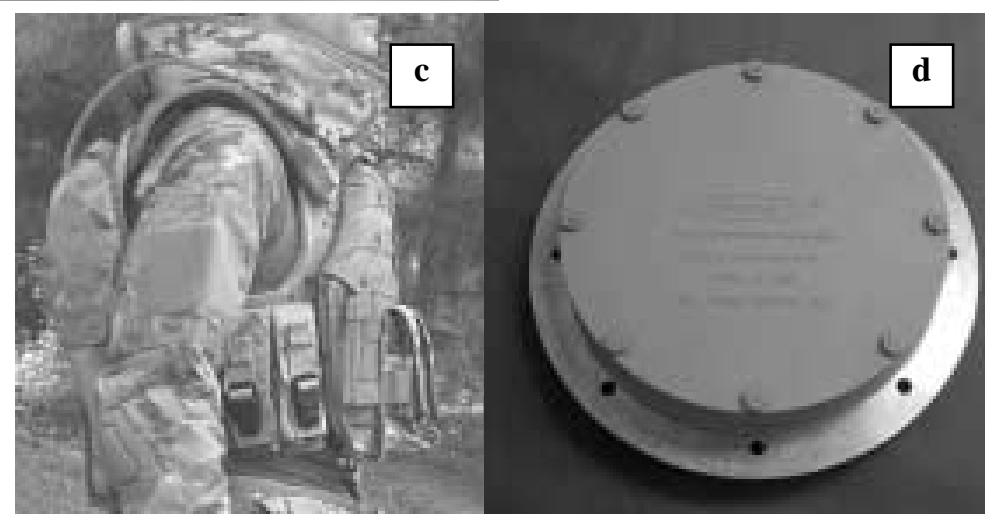

Fig. 3. Conformal broadband antennas mounted on (a) handset, (b) helmet, (c) body, and (d) platform.

An empirical theory on the fundamental bandwidth limitation for small antennas on platform is presented. For a lossless resonance antenna, it is limited by the combined electrical size of the antenna and its mounting platform according to the classical Chu theory. For a small conformable traveling wave antenna, as specified, mounted on a platform, there is no fundamental theoretical limitation on its octaval bandwidth. However, its lower bound of the operating bandwidth is limited by its electrical size, and its practical octaval bandwidth is finite, $<20$.

\section{REFERENCES}

[1] L. J. Chu, "Physical Limitations of Omnidirectional Antennas," J. Appl. Phys., Vol. 19, 1948.

[2] J. J. H. Wang, "A Critique and New Concept on Gain Bandwidth Limitation of Omnidirectional Antennas," Progress in Electromagnetics Research Symposium (PIERS) 2005, Hangzhou, China, August 2005.

[3] J. J. H. Wang, "The Spiral as a Traveling Wave Structure for Broadband Antenna Applications," Electromagnetics, 20-40, July-August 2000.

[4] J. J. H. Wang, "The Physical Foundation, Development History, and Ultra-wideband Performance of SMM (Spiral-Mode Microstrip) Antennas," 2005 IEEE Antennas and Prop. Symp., Washington, DC, July 2005.

[5] J. J. H. Wang and J. K. Tillery, "Broadband Miniaturized Slow-Wave Antenna," U.S. patent \#6,137,453, October 24, 2000; Taiwan patent \#138931, July 21, 2001; China patent \#191646, January 19, 2005.

[6] J. S. McLean, "A Re-Examination of the Fundamental Limits on the Radiation $Q$ of Electrically Small Antennas," IEEE Trans. Ant. Prop., May 1996.

[7] R. E. Collin, D. R. Rhodes, and G. V. Borgiotti, "Stored Energy, $Q$ and Frequency Sensitivity of Planar Aperture Antennas," IEEE Trans. Ant. Prop., July 1967.

[8] J. J. H. Wang, C. J. Stevens, and D. J. Triplett, "Ultra-wideband Omnidirectional Conformable Low-Profile Mode0 Spiral-Mode Microstrip (SMM) Antenna," 2005 IEEE Antennas and Prop. Symp., Washington, DC, July 2005.

[9] A. D. Yaghjian and S. R. Best, "Impedance, Bandwidth, and $Q$ of Antennas," IEEE Trans. Ant. Prop., April, 2005.

[10] J. J. H. Wang, "Broadband Omnidirectional Helmet Antennas," unpublished.

[11] J. J. H. Wang and D. J. Triplett, "A Multioctave Broadband Vest Antenna Based on SMM Antenna Technology," unpublished. 